\title{
Novel class of medications, orexin receptor antagonists, in the treatment of insomnia - critical appraisal of suvorexant
}

This article was published in the following Dove Press journal:

Nature and Science of Sleep

14 July 2016

Number of times this article has been viewed

\section{Jessica L Norman \\ Sarah L Anderson}

Department of Clinical Pharmacy, Skaggs School of Pharmacy and

Pharmaceutical Sciences, University of Colorado, Aurora, CO, USA
Correspondence: Sarah L Anderson Department of Clinical Pharmacy, Skaggs School of Pharmacy and Pharmaceutical Sciences, University of Colorado, Mail Stop C238, 12850 E Montview Boulevard, Room V20-2129, Aurora, CO 80045 , USA

$\mathrm{Tel}+\mathrm{I} 3037245926$

Fax + I 3037242627

Email sarah.anderson@ucdenver.edu
Abstract: Insomnia, a highly prevalent disorder, can be detrimental to patients' overall health and worsen existing comorbidities. Patients may have acute episodes of insomnia related to a traumatic event, but more commonly insomnia occurs chronically. While proper sleep hygiene and behavioral therapy play important roles in the nonpharmacologic management of shortterm and chronic insomnia, medications may also be required. Historically, insomnia has been treated with agents such as benzodiazepines, nonbenzodiazepine receptor agonists, and melatonin agonists. Dual orexin receptor antagonists represent a new class of medications for the treatment of insomnia, which block the binding of wakefulness-promoting neuropeptides orexin A and orexin B to their respective receptor sites. Suvorexant (Belsomra) is the first dual orexin receptor antagonist to be approved in the US and Japan and has demonstrated efficacy in decreasing time to sleep onset and increasing total sleep time. Its unique mechanism of action, data to support efficacy and safety over 12 months of use, and relative lack of withdrawal effects when discontinued may represent an alternative for patients with chronic insomnia who cannot tolerate or do not receive benefit from more traditional sleep agents. Suvorexant is effective and well tolerated, but precautions exist for certain patient populations, including females, obese patients, and those with respiratory disease. Suvorexant has only been studied vs placebo, and hence it is unknown how it directly compares with other medications approved by the US Food and Drug Administration for insomnia. Suvorexant is not likely to replace benzodiazepines or nonbenzodiazepine receptor antagonists as a first-line sleep agent but does represent a novel option for the treatment of patients with chronic insomnia.

Keywords: insomnia, orexin antagonist, suvorexant

\section{Introduction}

Insomnia is a prevalent condition that affects an estimated $6 \%-30 \%$ of the adult population. ${ }^{1}$ The International Classification of Sleep Disorders, third edition, describes insomnia as 1) a report of sleep initiation or maintenance problems, 2) adequate opportunity and circumstances to sleep, and 3) daytime consequences. One or a combination of these issues occurs at least three times per week and will be ongoing for 3 months. $^{2}$ Patients with insomnia have traditionally relied on benzodiazepines, nonbenzodiazepine receptor agonists, or melatonin agonists as primary therapeutic categories to treat insomnia (Table 1). While these medications are often effective, they may not be efficacious or appropriate for every patient, especially when used chronically. Innovative therapeutic agents to treat insomnia have been highly sought after to meet this need. 
Table I Selected pharmacokinetic parameters of US FDAapproved medications for insomnia treatment

\begin{tabular}{|c|c|c|c|}
\hline Drug & Dose (mg) & $\mathbf{T}_{\max }$ (hours) & $t_{1 / 2}$ (hours) \\
\hline \multicolumn{4}{|c|}{ Benzodiazepine receptor antagonists } \\
\hline Estazolam & $0.5-2$ & 2 & $10-24$ \\
\hline Flurazepam & $15-30$ & $0.5-1$ & 74-90 \\
\hline Lorazepam & $2-4$ & 2 & 12 \\
\hline Quazepam & $7.5-15$ & 2 & 39 \\
\hline Temazepam & $7.5-30$ & $1.2-1.6$ & $3.5-18.4$ \\
\hline Triazolam & $0.125-0.5$ & 2 & $1.5-5.5$ \\
\hline \multicolumn{4}{|c|}{ Nonbenzodiazepine receptor antagonists } \\
\hline Eszopiclone & $1-3$ & $\mathrm{I}-\mathrm{I} .5$ & 6 \\
\hline Zaleplon & $5-20$ & I & I \\
\hline Zolpidem & $5-10$ & 1.6 & $2-2.6$ \\
\hline Zolpidem ER & $6.25-12.5$ & 1.6 & $2-2.6$ \\
\hline Zolpidem SL & $1.75-3.5$ & 1.6 & $2-2.6$ \\
\hline Zolpidem SL (Canada) & $5-10$ & 1.6 & $2-2.6$ \\
\hline \multicolumn{4}{|c|}{ Melatonin receptor agonist } \\
\hline Ramelteon & 8 & $0.5-1.5$ & $\mathrm{I}-2.6$ \\
\hline \multicolumn{4}{|l|}{ Tricyclic antidepressant } \\
\hline Doxepin & $3-6$ & 3.5 & 15.3 \\
\hline \multicolumn{4}{|c|}{ Orexin receptor antagonist } \\
\hline Suvorexant & $10-20$ & 2 & 12 \\
\hline
\end{tabular}

Abbreviations: FDA, Food and Drug Administration; ER, extended release; SL, sublingual; $\mathrm{T}_{\max }$, median time to maximum concentration; $\mathrm{t}_{1 / 2}$, terminal half-life.

Dual orexin receptor antagonists (DORAs) are a novel therapeutic class of medications for the treatment of insomnia. Suvorexant is the first agent to be approved in this new class and gained US Food and Drug Administration (FDA) approval in 2014. ${ }^{3}$ It is also the first insomnia medication in a new class since ramelteon was approved in 2005. Orexins (also called hypocretins) are neuropeptides that stimulate wake-promoting systems in the sleep-wake cycle. First discovered in narcolepsy research in the early 1990s, orexins became a natural target for the development of therapeutic compounds for the treatment of insomnia. ${ }^{4}$

\section{Pharmacology Mechanism of action}

Suvorexant highly selectively blocks neuropeptides orexin A and orexin B from binding to the orexin receptor types 1 and 2 (OX1R and OX2R), respectively, suppressing wakefulness. Both OX1R and OX2R are located in regions of the brain responsible for promoting arousal and vigilance and follow a circadian pattern in which orexin signaling increases during periods of wakefulness and decreases during sleep. ${ }^{5,6}$ Preclinical and clinical studies have demonstrated that blocking orexin receptors increases time spent in all stages of sleep and does not shift the sleep profile; therefore, DORAs such as suvorexant may promote better sleep in chronic insomnia. ${ }^{7}$ Additionally, preclinical studies show that orexin receptors require $\sim 65 \%-80 \%$ occupancy by a receptor antagonist in order to promote sleep. The coupling of the circadian pattern of orexin signaling with the requirement for high receptor occupancy to promote sleep initially led researchers to hypothesize that suvorexant more easily blocks orexin-mediated arousal during sleep and its pharmacologic effects lessen as the body resumes wakefulness and orexin signaling in the brain increases. ${ }^{8}$ This balance in orexin receptor activity and drug action was proposed to optimize the action of suvorexant when needed and wearing off when its effects were no longer warranted, making it an ideal insomnia treatment. However, suvorexant binds to and releases from the orexin receptor very slowly, creating residual pharmacologic effects beyond that predicted by the pharmacokinetics (PK) of the agent. ${ }^{6}$

\section{Pharmacodynamics}

The suvorexant pharmacodynamic parameter evaluated in a clinical trial and reported in the prescribing information is its effect on the corrected QT interval (QTc) interval. Healthy subjects $(\mathrm{N}=53)$ were randomized in a placebo- and active-controlled (moxifloxacin) crossover study. Subjects received moxifloxacin $400 \mathrm{mg}$ or up to $240 \mathrm{mg}$ of suvorexant, and no appreciable QTc prolongation was noted in patients receiving suvorexant. ${ }^{3}$

\section{Pharmacokinetics}

Suvorexant PK have been characterized in adults with and without insomnia. In healthy young men, suvorexant peak concentrations $\left(\mathrm{T}_{\max }\right)$ occurred at 180 minutes and had a terminal half-life $\left(\mathrm{t}_{1 / 2}\right)$ of $9-13$ hours. ${ }^{9}$ Pooled data of all patients show a slightly quicker time to peak concentration with a median $\mathrm{T}_{\max }$ of 120 minutes (range 30-360 minutes) and a similar $t_{1 / 2}$ of 12 hours. ${ }^{3}$ Food ingestion can delay the $\mathrm{T}_{\max }$ by 90 minutes, so suvorexant should not be administered with or soon after a meal if faster sleep onset is preferred.

Suvorexant is metabolized through cytochrome P450 (CYP) 3A4 (major) and CYP219 (minor). Hydroxyl suvorexant, the metabolite of suvorexant, is not therapeutically active. Elimination occurs through feces $(66 \%)$ and urine $(23 \%) .^{3}$

\section{Efficacy and tolerability}

Suvorexant has been studied for the treatment of primary insomnia in one Phase $\mathrm{II}^{10}$ and three Phase III trials. ${ }^{11,12}$ The Phase II trial was a randomized, double-blind, placebocontrolled crossover study, and the Phase III trials were each randomized, double-blind, placebo-controlled trials. Phase III trial results are summarized in Table 2. The Phase II and Phase III trials ranged from 1 month to 1 year in duration, were conducted in multiple countries and were industry sponsored. 
Table 2 Suvorexant Phase III clinical trial data

\begin{tabular}{|c|c|c|c|c|c|c|c|c|c|c|}
\hline Study & Intervention & $\mathbf{N}$ & $\begin{array}{l}\text { Female } \\
(\%)\end{array}$ & $\begin{array}{l}N \\
<65 \text { years } \\
(\%)\end{array}$ & $\begin{array}{l}\text { Baseline } \\
\text { sTST } \\
\text { (minutes) }\end{array}$ & $\begin{array}{l}\text { Baseline } \\
\text { sTSO } \\
\text { (minutes) }\end{array}$ & $\begin{array}{l}\text { Change in } \\
\text { sTST at } \\
\text { month } 3 \\
\text { (minutes) }\end{array}$ & $\begin{array}{l}\text { Change in } \\
\text { sTSO at } \\
\text { month } 3 \\
\text { (minutes) }\end{array}$ & $\begin{array}{l}\text { Change in } \\
\text { sTST at } \\
\text { month I2 } \\
\text { (minutes) }\end{array}$ & $\begin{array}{l}\text { Change in } \\
\text { sTSO at } \\
\text { month } 12 \\
\text { (minutes) }\end{array}$ \\
\hline \multirow{5}{*}{$\begin{array}{l}\text { Herring } \\
\text { et al," trial I }\end{array}$} & Suvorexant & 254 & $162(63.8)$ & I47 (57.9) & 322 & 63 & $10.7^{*}$ & $-5.2^{*}$ & - & - \\
\hline & $20 / 15$ mg & & & & & & & & & \\
\hline & Suvorexant & 383 & $230(60.1)$ & $222(58)$ & 316 & 68 & $19.7^{*}$ & $-8.4^{*}$ & - & - \\
\hline & $30 / 40 \mathrm{mg}$ & & & & & & & & & \\
\hline & Placebo & 384 & $245(63.8)$ & $223(58.1)$ & 316 & 67 & - & - & - & - \\
\hline \multirow{5}{*}{$\begin{array}{l}\text { Herring } \\
\text { et al," trial } 2\end{array}$} & Suvorexant & 239 & $157(65.7)$ & $144(60.3)$ & 298 & 86 & $22.1 *$ & $-7.6 *$ & - & - \\
\hline & $20 / 15 \mathrm{mg}$ & & & & & & & & & \\
\hline & Suvorexant & 387 & 267 (69) & $229(59.2)$ & 315 & 74 & $25 . I^{*}$ & $-13.2^{*}$ & - & - \\
\hline & $30 / 40 \mathrm{mg}$ & & & & & & & & & \\
\hline & Placebo & 383 & $247(64.5)$ & $226(59)$ & 310 & 81 & - & - & - & - \\
\hline \multirow{3}{*}{$\begin{array}{l}\text { Michelson } \\
\text { et } \mathrm{al}^{12}\end{array}$} & Suvorexant & 521 & $287(55)$ & $2 \mid 3(4 \mid)$ & 320.4 & 65.9 & - & - & $27.5^{*}$ & $-9.7^{*}$ \\
\hline & $40 / 30 \mathrm{mg}$ & & & & & & & & & \\
\hline & Placebo & 238 & I49 (58) & $107(42)$ & 330.1 & 64.9 & - & - & - & - \\
\hline
\end{tabular}

Notes: ${ }^{*} P<0.05$. Doses listed are for nonelderly/elderly study participants.

Abbreviations: sTSO, subjective time to sleep onset; sTST, subjective total sleep time.

The primary and secondary efficacy outcomes varied among studies and included patient-reported subjective total sleep time (sTST), subjective time to sleep onset (sTSO), subjective wake after sleep onset (sWASO), subjective number of awakenings (sNAW), subjective quality of sleep (sQUAL), subjective refreshed feeling on waking (sFRESH), and latency to onset of persistent sleep (LPS). The Diagnostic and Statistical Manual of Mental Disorders, fourth edition, text revision criteria were used for the diagnosis of primary insomnia in all studies. Patients were excluded if they were not in good mental or physical health (ie, had other sleep disorders, confounding neurologic disorders, current major affective or psychiatric illness, substance abuse, or an unstable medical condition).

The Phase II trial by Herring et a ${ }^{10}$ evaluated four doses of suvorexant (10 mg, $20 \mathrm{mg}, 40 \mathrm{mg}$, and $80 \mathrm{mg}$ ) in a two-period crossover polysomnography (PSG) study. Patients received suvorexant $10 \mathrm{mg}(\mathrm{n}=62), 20 \mathrm{mg}(\mathrm{n}=61), 40 \mathrm{mg}(\mathrm{n}=59)$, or $80 \mathrm{mg}(\mathrm{n}=61)$ in one 4-week period and placebo $(\mathrm{n}=249)$ in the other 4-week period. There was a single-blind, 1-week placebo washout between periods. The coprimary efficacy outcomes were sleep efficiency (SE) (defined as TST divided by time in bed in minutes [fixed at 480 for this study] multiplied by 100 ) on night 1 and at the end of week 4 . The secondary outcomes were WASO (defined as duration of wakefulness after persistent sleep onset to lights on) and LPS (defined as the duration of time from lights off to persistent sleep onset). Baseline characteristics, including sex, race, age, and body mass index (BMI), were similar among treatment groups. Patients had an average SE of $66 \%$, a TST of 316 minutes, a WASO of 101 minutes, and an LPS of 69 minutes at baseline. The average Insomnia Severity Index (ISI) total score at baseline was 17, which corresponds to moderate severity clinical insomnia, and the average Sheehan Disability Scale total score at baseline was 9, which corresponds to mild disruption of work/social/family life by disease symptoms. The baseline symptom severity was similar between groups.

All doses of suvorexant were more effective than placebo (all $P$-values $<0.01$ ) in improving the coprimary endpoints of SE on night 1 and at the end of week 4, with a dose-response effect observed. The SE on night 1 was $5.2 \%$ (95\% CI 1.9-8.6), 7.6\% (95\% CI 4.2-11.0), 10.8\% (95\% CI 7.4-14.2), and $12.9 \%$ (95\% CI 9.5-16.3) for suvorexant $10 \mathrm{mg}, 20 \mathrm{mg}$, $40 \mathrm{mg}$, and $80 \mathrm{mg}$, respectively. The SE at the end of week 4 was $4.7 \%$ (95\% CI $1.6-7.8), 10.4 \%$ (95\% CI $7.2-13.6$ ), $7.8 \%$ (95\% CI $4.6-10.9)$, and $7.6 \%$ (95\% CI 4.4-10.9) for suvorexant $10 \mathrm{mg}, 20 \mathrm{mg}, 40 \mathrm{mg}$, and $80 \mathrm{mg}$, respectively. Dose-related effects were also observed for LPS and WASO $(P \leq 0.001)$. Although the results suggested that the $40 \mathrm{mg}$ and $80 \mathrm{mg}$ doses had greater effects on efficacy outcomes than the $10 \mathrm{mg}$ and $20 \mathrm{mg}$ doses, formal testing of dose-response was not performed.

The $10 \mathrm{mg}$ and $20 \mathrm{mg}$ suvorexant doses had an adverse event rate similar to placebo, whereas the $40 \mathrm{mg}$ and $80 \mathrm{mg}$ doses showed an increased rate of adverse events. The most common patient-reported adverse effects were somnolence (drowsiness), headache, dizziness, and abnormal dreams. Other common adverse effects included upper respiratory infection, urinary tract infection, and increased alanine aminotransferase. No significant evidence of rebound 
insomnia, withdrawal, or morning-after residual effects was detected when patients discontinue suvorexant. Patients who continued in the 3-month extension phase reported less somnolence, indicating a degree of tolerance to the adverse effects of suvorexant.

Herring et al assessed suvorexant efficacy in two 3-month trials in nonelderly (18-64 years) and elderly ( $\geq 65$ years) patients. ${ }^{11}$ Trial 1 included an optional 3-month double blind extension. Suvorexant doses of 40/30 mg (40 mg for nonelderly patients and $30 \mathrm{mg}$ for elderly patients) and $20 / 15 \mathrm{mg}$ (20 mg for nonelderly patients and $15 \mathrm{mg}$ for elderly patients) were evaluated among 1,021 patients in trial 1 and 1,009 patients in trial 2. Both trials included a 1-week, randomized, double-blind, run-out period after the double-blind treatment to assess for withdrawal and rebound insomnia. The primary endpoints included the change from baseline at 1 month and 3 months for subjective (obtained from sleep diary: sTST, sTSO, sWASO, sNAW, sQAUL, and sFRESH) and objective (obtained from PSG: LPS and WASO) measures. The two trials randomized 770 patients to the suvorexant $40 / 30 \mathrm{mg}$ group, 493 patients to the suvorexant 20/15 mg group, and 767 patients to the placebo group. The baseline average ISI score was 16-17 in all treatment groups, which corresponds to moderate severity clinical insomnia. Baseline patient characteristics and symptom severity were similar among treatment groups.

Suvorexant 40/30 mg was superior to placebo in improving sleep maintenance, measured by sTST and WASO, at month 1 and month 3 in both trials $(P<0.001$ for all measures). The change from baseline in sTST at month 3 was 19.7 minutes (95\% CI 11.9-27.6) and 25.1 minutes (95\% CI 16.0-34.2) for trials 1 and 2, respectively. The change from baseline in WASO at month 3 was -22.9 minutes $(95 \% \mathrm{CI}$ -30.3 to -15.4$)$ and -29.4 minutes $(95 \% \mathrm{CI}-36.7$ to -22.1$)$ for trials 1 and 2, respectively. Suvorexant 40/30 mg was also superior to placebo in improving sleep onset at month 1 and month 3 , measured by sTSO ( $P<0.01$ for both) and LPS $(P<0.001$ for both) in trial 1 . The change from baseline at month 3 was -8.4 minutes $(95 \% \mathrm{CI}-12.8$ to -4.0$)$ for sTSO and -9.4 minutes $(95 \% \mathrm{CI}-14.6$ to -4.3$)$ for LPS in trial 1. However, the treatment difference in LPS at month 3 was not significant in trial 2. In addition, the subjective measurements of sWASO and sQUAL were significant at month 3.

Suvorexant 20/15 mg was superior to placebo in improving sleep maintenance, measured by sTST and WASO at month 1 and month 3 in both trials $(P<0.001$ for all measures). The change from baseline in sTST at month 3 was 10.7 minutes (95\% CI 1.9-19.5) and 22.1 minutes (95\% CI
11.5-32.6) for trials 1 and 2, respectively. The change from baseline in WASO at month 3 was -16.6 minutes $(95 \% \mathrm{CI}$ -24.8 to -8.3 ) and -31.1 minutes (95\% CI -40.1 to -22.2$)$. It was superior to placebo for improving sleep onset, measured by LPS, at month 1 but did not significantly differ from placebo on sTSO at any time point.

A predefined improvement of $\geq 6$ points on the ISI score was deemed a treatment response. Both the suvorexant 40/30 mg and 20/15 mg groups demonstrated significantly higher proportions of patients achieving a meaningful treatment response compared with placebo $(P<0.01$ for all comparisons); however, there was a substantial placebo effect. Approximately 40\% (39.3\% in trial 1 and $45.2 \%$ in trial 2) of patients receiving placebo experienced $a \geq 6$ point improvement in ISI score.

There were similar incidences of adverse effects and discontinuations due to adverse effects in the suvorexant and placebo groups. The most common adverse effect in the suvorexant group was somnolence (trial 1: suvorexant $20 / 15 \mathrm{mg}=5.1 \%$, suvorexant $40 / 30 \mathrm{mg}=10.7 \%$, and placebo $=3.4 \%$; trial 2: suvorexant $20 / 15 \mathrm{mg}=8.4 \%$, suvorexant $40 / 30 \mathrm{mg}=10.3 \%$, and placebo $=3.1 \%$ ), which appeared to follow a dose-response relationship. While not statistically significant, the proportions of patients meeting criteria for rebound insomnia in trial 1 were greater in the cohort switching from suvorexant to placebo than in the cohort remaining on placebo. In trial 2 , the proportions of patients meeting criteria for rebound insomnia were statistically significantly greater in patients switching from suvorexant $40 / 30 \mathrm{mg}$ to placebo than in patients switching from placebo to placebo. There was no evidence of withdrawal in trial 1 based on the Tyrer Withdrawal Symptom Questionnaire. In trial 2, statistically significantly more patients switching from suvorexant $40 / 30 \mathrm{mg}$ to placebo met withdrawal criteria on night 1 than patients remaining on suvorexant $40 / 30 \mathrm{mg}$, an effect that was not observed on the following two nights. No statistically significant differences were found between suvorexant and placebo in terms of next-morning residual effects. The authors concluded that the magnitude of rebound and withdrawal effects was small and likely to be clinically insignificant for most patients.

In conclusion, the two 3-month trials by Herring et $\mathrm{al}^{11}$ demonstrated that suvorexant remains effective over a 3-month period, the effects of suvorexant on sleep maintenance are more pronounced than for sleep onset, and suvorexant $40 / 30 \mathrm{mg}$ is modestly more effective than suvorexant 20/15 mg for most outcomes and time points. 
Somnolence may lessen the longer patients are treated with suvorexant.

Michelson et al assessed suvorexant safety and efficacy for 1 year. ${ }^{12}$ Patients were randomly assigned to receive suvorexant (40 mg for patients $<65$ years old and $30 \mathrm{mg}$ for patients $\geq 65$ years old) or placebo at a $2: 1$ ratio. The primary objective was to assess the safety and tolerability of suvorexant at 1 year. The secondary objectives were to assess the efficacy for sleep maintenance (sTST) and sleep onset (sTSO) over the first month of treatment. At the end of 1 year of treatment, there was a subsequent 2-month randomized discontinuation phase in which patients on suvorexant either continued suvorexant or were abruptly switched to placebo. The discontinuation phase was an exploratory study with a primary endpoint of relapse prevention where relapse was defined as a return to moderate or severe insomnia (ISI $>14$ ).

Of the 1,076 patients who were screened for inclusion in the study, 522 patients were randomly assigned to the suvorexant group and 259 patients were assigned to the placebo group. The mean ISI score was 14.5 in the suvorexant group and 13.7 in the placebo group at baseline, corresponding to subthreshold insomnia. Other baseline patient characteristics and symptom severity were similar between treatment groups. A total of 484 patients completed the 1-year phase, with 166 patients randomized to the suvorexant-placebo group, 156 patients randomized to the suvorexant-suvorexant group, and 162 patients randomized to the placebo-placebo group. Sixty-two percent of patients in the suvorexant group and $63 \%$ of patients in the placebo group completed the 1-year study. Throughout the study, $69.5 \%$ of patients in the suvorexant group and $63.6 \%$ of patients in the placebo group experienced at least one adverse event (difference $=5.9 ; 95 \% \mathrm{CI}-1.1$ to -13.1 ). Similar proportions of patients in the suvorexant group and placebo group experienced a serious drug-related adverse event $(0.2 \%$ vs $1.2 \%$; difference $=-1.0 ; 95 \% \mathrm{CI}-3.2$ to $-0.1)$ and discontinued the treatment medication due to an adverse event $(11.7 \%$ vs $8.5 \%$; difference $=3.2 ; 95 \%$ $\mathrm{CI}-1.5$ to -7.4$)$. The most common adverse effects were somnolence, fatigue, and dry mouth. Somnolence was most common in the first 3 months $(11 \%$ vs $2 \%)$ and was less likely in the second 3 months $(3 \%$ vs $<1 \%)$ for suvorexant vs placebo, respectively.

At 1 month, when compared with placebo, suvorexant improved sTST (40.9 minutes vs 17.5 minutes, respectively; $P<0.0001)$ and sTSO $(-19.2$ minutes vs -9.0 minutes, respectively; $P=0.0004)$. Using the sTST $20 \%$, sTST
$10 \%$, and sTST 5\% responder definitions, risk for relapse in the suvorexant-placebo group was greater than in the suvorexant-suvorexant group. However, using the ISI definition, the difference in risk for relapse between the groups was not statistically significant. There was no significant difference between the suvorexant group and placebo group in terms of adverse events upon discontinuation of the study drug. While not statistically significant, the proportions of patients with rebound insomnia were greater in the suvorexant-placebo group when compared with the placebo-placebo group. There was no evidence of withdrawal when comparing the suvorexant-suvorexant group with the suvorexant-placebo group. This study represents the first to evaluate a chronically used hypnotic agent for 1 year. The only other randomized, double-blind, placebo-controlled trial to evaluate the use of a hypnotic for this length of time found the incidence of rebound insomnia in patients receiving zolpidem for 12 months with placebo interruptions for a week at months 1,4 , and $12 .^{13}$

Kishi et $\mathrm{al}^{14}$ summarized the four trials described earlier in a systematic review and meta-analysis. The meta-analysis included 3,076 patients, and the mean age of the patients was 56.6 years, of which $38.2 \%$ were male and $77.0 \%$ were white. The primary efficacy endpoints were sTST and sTSO at 1 month. The secondary outcomes included sTST and sTSO at 1 week and 3 months; sWASO, sQAUL, and sNAW at 1 week, 1 month, and 3 months; LPS and WASO at 1 day, 1 month, and 3 months, sFRESH at 1 month and 3 months; discontinuation rate; and a variety of patient and provider questionnaires. Suxorexant was superior to placebo with regard to all primary (sTST: $-20.16,95 \% \mathrm{CI}-25.01$ to $-15.30,1,889$ patients, and three trials; sTSO: $-7.62,95 \%$ $\mathrm{CI}-11.03$ to $-4.21,1,889$ patients, and three trials) and secondary outcomes with the exceptions of sQUAL at 1 week, sWASO and LPS at 3 months, and sNAW at all time points. There was a higher incidence of abnormal dreams, somnolence, excessive daytime sleepiness/sedation, fatigue, and dry mouth in the suvorexant group than in the placebo group. There was more rebound insomnia (defined by decrease in sTST) at day 1, day 3, and days 1-3 in the group switching from suvorexant to placebo than in the group switching from placebo to placebo. There was no difference in regard to withdrawal symptoms and rebound insomnia (defined by increase in STSO) at day 1 , day 2 , day 3 , and days $1-3$ between suvorexant and placebo.

Another systematic review of suvorexant Phase II and III randomized controlled trials concluded that the number needed to treat vs placebo for at least a 6-point improvement 
on the ISI scale after 3 months of treatment was $8(95 \% \mathrm{CI}$ 6-14) for suvorexant $15 \mathrm{mg}, 20 \mathrm{mg}, 30 \mathrm{mg}$, and $40 \mathrm{mg} .{ }^{15}$ Based on the most commonly occurring adverse event (somnolence), the number needed to harm vs placebo was 13 (95\% CI 11-18) for suvorexant 30/40 mg and 28 (95\% CI 17-82) for suvorexant $15 / 20 \mathrm{mg}$. Rebound insomnia and withdrawal symptoms were not noted after 3 months or 12 months of suvorexant therapy. The number needed to treat and number needed to harm data were similar for patients $>65$ years old compared with those $\leq 65$ years old. These data are comparable with treatment and harm estimates for other sedative hypnotic agents. ${ }^{16,17}$

\section{Safety considerations}

Suvorexant has been well-tolerated in clinical trials but has several precautions and a specific contraindication to note. Special safety concerns related to female sex, obese patients, and those with respiratory disease also exist.

\section{Contraindications}

The only population specifically contraindicated for the use of suvorexant is patients with narcolepsy as the condition will be exacerbated with suvorexant use. While not contraindicated, suvorexant use is not recommended in patients receiving strong CYP3A inhibitors (eg, ketoconazole, clarithromycin, and ritonavir). ${ }^{3}$

\section{Adverse events}

\section{General}

The most common adverse event experienced by patients who received suvorexant during clinical trials was somnolence ( $7 \%$ vs $3 \%$ for placebo). Other commonly reported adverse events ( $\geq 2 \%$ incidence and greater than placebo) in patients who received suvorexant for 3 months were diarrhea, xerostomia, upper respiratory tract infections, headache, dizziness, abnormal dreams, and cough. ${ }^{11}$ In an extended study in which patients received suvorexant treatment for up to 12 months, the most frequent adverse events were similar to those previously described. ${ }^{12}$

Dose-related adverse effects that led to the FDA requesting the drug manufacturer to decrease the starting dose of suvorexant included excessive daytime impairment, additive central nervous system depression when coadministered with other central nervous system depressants, abnormal thinking and behavioral changes, worsening depression or increases in suicidal ideation, sleep paralysis, hypnagogic and hypnopompic hallucinations, and cataplexy-like symptoms. $^{7}$

\section{Special population considerations}

\section{Female patients}

PK data in female patients receiving suvorexant showed an increased area under the curve (AUC) and maximum serum concentration $\left(\mathrm{C}_{\text {max }}\right)$ when compared with male patients. When female patients were administered a $40 \mathrm{mg}$ dose of suvorexant, the AUC was increased by $17 \%$ and the $C_{\max }$ was increased by $9 \%$ compared to male patients receiving the same dose. ${ }^{3}$ In clinical trials, female patients receiving suvorexant $15 \mathrm{mg}$ and $20 \mathrm{mg}$ experienced somnolence more often than male patients receiving the same dose ( $8 \%$ females vs $3 \%$ males; no $P$-value reported). Also, the side effects of headache, abnormal dreams, xerostomia, cough, and upper respiratory tract infection were more commonly observed in female patients. ${ }^{3,7}$ Two safety studies conducted in healthy elderly and nonelderly patients aimed to assess nighttime administration of suvorexant and its next-day effects on driving performance found that four female patients receiving suvorexant stopped their driving test prematurely due to somnolence. This result was not replicated in males. Because of this issue observed during the safety study, it is recommended that patients receiving suvorexant $20 \mathrm{mg}$ be warned about the next-day driving impairment. ${ }^{3}$ Despite these sex differences in suvorexant exposure, there is no female sex-specific dosing recommendation other than to use caution in the female population.

\section{Obese patients}

PK studies concluded that compared to nonobese patients $\left(\right.$ BMI $\left.<25 \mathrm{~kg} / \mathrm{m}^{2}\right)$, obese patients $\left(\right.$ BMI $>30 \mathrm{~kg} / \mathrm{m}^{2}$ ) were exposed to $15 \%$ more drug for 9 hours after a $20 \mathrm{mg}$ dose of suvorexant. Both the $\mathrm{AUC}$ and $C_{\max }$ are increased in female patients and obese patients separately, and these increases are compounded in obese female patients. However, analogous to the absence of sex-specific dosing recommendations, there are no dosing recommendations related to weight or $\mathrm{BMI}^{3}$

\section{Respiratory disease}

A recently published randomized, double-blind, placebocontrolled, crossover study in which 26 patients with mild or moderate obstructive sleep apnea were randomized to receive either suvorexant $40 \mathrm{mg}$ or placebo and then crossed over into the other treatment found a small increase in the apnea-hypopnea index. Although the dose used was twice that of the FDA-approved dose and increase found was deemed to be not clinically important, there was high interand intrapatient variabilities during both study phases such that clinically relevant concerns related to use of suvorexant 
in patients with obstructive sleep apnea cannot be ruled out based on these data. ${ }^{18}$

\section{Renal impairment}

Suvorexant has been evaluated in patients with renal impairment. In one study, eight male and female patients with renal impairment (estimated creatinine clearance $=30 \mathrm{~mL} / \mathrm{min}$ ) were matched with eight healthy male and female subjects (estimated creatinine clearance $=80 \mathrm{~mL} / \mathrm{min}$ ) and each received a one-time dose of suvorexant $20 \mathrm{mg}$. After the one-time dose of suvorexant, there was no difference in AUC between groups $(90 \%$ CI $1.18 ; 0.83,1.67)$. There was a $\mathrm{T}_{\max }$ of 2 hours in the renally impaired group compared with 1 hour in the healthy group; the $t_{1 / 2}$ was 13.5 hours in both groups. ${ }^{3}$ The differences in PK parameters were deemed nonclinically significant, and there were no renal dose adjustments recommended for patients with renal impairment receiving suvorexant.

\section{Hepatic impairment}

An analogous study in which eight hepatically impaired patients with a Child-Pugh score of 7-9 were matched with eight healthy male and female subjects and each received a one-time dose of suvorexant $20 \mathrm{mg}$ also demonstrated similar AUC, $\mathrm{t}_{1 / 2}$, and $C_{\text {max }}$ data to that seen in patients with renal impairment. ${ }^{3}$ Based on these findings, patients with mild to moderate hepatic impairment do not require dose adjustments of suvorexant. ${ }^{3}$ Suvorexant has not been studied in patients with severe hepatic impairment and is therefore not recommended for use in this population.

\section{Drug-drug interactions}

\section{Drug effects on suvorexant}

Suvorexant was coadministered with ketoconazole, diltiazem, and rifampin during clinical studies. Coadministration of suvorexant with ketoconazole and diltiazem resulted in a higher systemic exposure to suvorexant. Concomitant therapy with ketoconazole and suvorexant resulted in threefold increase in AUC and an extended $t_{1 / 2}$ of 19.4 hours. ${ }^{3}$ When a single dose of suvorexant $20 \mathrm{mg}$ was administered with multiple daily doses of diltiazem $240 \mathrm{mg}$, the AUC increased approximately twofold and the $t_{1 / 2}$ increased to 16.1 hours. Because the PK changes were more intense with ketoconazole (a strong CYP3A4 inhibitor) than diltiazem (a moderate CYP3A4 inhibitor), the manufacturer of suvorexant recommends avoiding use in patients receiving "strong" CYP3A4 inhibitors and to use a reduced starting dose of $5 \mathrm{mg}$ in patients concurrently receiving "moderate" CYP3A4 inhibitors. ${ }^{3}$
Concomitant use of rifampin and suvorexant was studied in patients who received a single dose of suvorexant $40 \mathrm{mg}$ and multiple doses of rifampin $600 \mathrm{mg}$. Concomitant use of these therapies substantially decreased the $t_{1 / 2}$ to 7.7 hours and produced an $87 \%$ decrease in AUC. ${ }^{3}$ The shortened $t_{1 / 2}$ and substantial reduction in suvorexant exposure with concomitant rifampin use likely make suvorexant ineffective for treating insomnia in a patient receiving both medications.

A study of 31 healthy adults who received suvorexant $40 \mathrm{mg}$ and either placebo solution of $0.7 \mathrm{~g} / \mathrm{kg}$ of alcohol demonstrated that suvorexant in combination with alcohol increased reaction time vs either alone and had additive negative effects on sustained attention, working memory, and postural stability. There was no PK interaction noted. Based on the additive psychomotor effects when suvorexant was combined with alcohol, the authors recommend that alcohol should be avoided in patients receiving suvorexant. ${ }^{19}$

\section{Effects of suvorexant on other drugs}

Suvorexant has been coadministered with midazolam, combined oral contraceptives (ethinyl estradiol/norgestimate), warfarin, and digoxin, and has caused no meaningful interactions with any of these medications. ${ }^{3}$ However, due to the slight increase in digoxin concentrations that occurred with coadministration and the narrow therapeutic index observed with digoxin, monitoring of digoxin concentrations is recommended in patients receiving concomitant suvorexant. ${ }^{3}$ Recently published in vitro and in vivo characterization of the metabolism of suvorexant confirmed that suvorexant is a reversible inhibitor of CYP3A4 and 2C19 and a weak inducer of CYP3A4, 1A2, and 2B6. The authors concluded that the low plasma concentrations of the drug at therapeutic doses make it unlikely that suvorexant would cause any significant drug interactions related to its inhibition or induction of CYP enzymes. $^{20}$

\section{Abuse potential}

Suvorexant, as with benzodiazepines and nonbenzodiazepine receptor antagonists, is a schedule $\mathrm{C}$-IV controlled substance because it can be abused or cause physical dependence. The abuse potential of high-dose suvorexant ( $40 \mathrm{mg}, 80 \mathrm{mg}$, and $150 \mathrm{mg}$ ) was compared with that of high-dose zolpidem (15 $\mathrm{mg}$ and $30 \mathrm{mg}$ ) in a Phase I study of 36 healthy males and females who were recreational polydrug users with a history of sedative and psychedelic drug use. ${ }^{21}$ Resullts were comparable between suvorexant and zolpidem regarding subjects" "drug liking" scores over time on the visual analog scale compared to placebo. Differences were noted in several 
secondary objectives, however, including a flat dose-response profile for suvorexant that was not observed with zolpidem and fewer perceptual changes with suvorexant compared with zolpidem. The safety implications of the latter finding are that users of suvorexant have the potential to abuse the medication but are less likely to harm themselves. These secondary findings of lower rates of perceptual changes and hallucinations confirmed the findings from the Phase III trial by Michelson et al, which demonstrated that the adverse effects of derealization, euphoria, hallucination, and depersonalization were similar between the suvorexant and placebo groups. ${ }^{12}$ Postmarketing experience with suvorexant will be important for further evaluating its abuse potential, but to date it appears to confer no more risk than traditional sleep agents, such as zolpidem.

\section{Dosing}

As described, suvorexant was studied at doses ranging from $10 \mathrm{mg}$ to $80 \mathrm{mg}$ in the Phase II clinical trial and at doses between $15 \mathrm{mg}$ and $40 \mathrm{mg}$ in Phase III clinical trials. However, adverse events experienced by patients at doses $>20 \mathrm{mg}$, such as next-day drowsiness and risk of car accidents, led the FDA to approve suvorexant at doses lower than doses studied (5 mg, $10 \mathrm{mg}, 15 \mathrm{mg}$, and $20 \mathrm{mg}$ ). The labeled starting dose for most patients is $10 \mathrm{mg}$, which was not studied in Phase III clinical trials. In patients concurrently receiving a moderate CYP3A inhibitor (eg, atazanavir, diltiazem, and verapamil), the starting dose is $5 \mathrm{mg}$, a dose which was not studied in either Phase II or Phase III clinical trials. Patients initiated on $5 \mathrm{mg}$ or $10 \mathrm{mg}$ can have their dose increased to $10 \mathrm{mg}$ or $20 \mathrm{mg}$, respectively, if the starting dose is ineffective.

\section{Place in therapy}

Because of its longer half-life, suvorexant is indicated for the treatment of both sleep onset and sleep maintenance insomnia. Suvorexant has a novel mechanism of action and, therefore, may be useful in patients who do not tolerate or do not respond to traditional sleep agents, such as benzodiazpines (eg, temazepam), nonbenzodiazepine receptor agonists (eg, zolpidem), melatonin receptor agonists (ie, ramelteon), and tricyclic antidepressants (ie, doxepin). Data from recent meta-analyses show the efficacy and effect size of suvorexant appears to be similar to ramelteon, benzodiazepines, and antidepressants. ${ }^{14,22,23}$ Furthermore, a Phase II trial found that suvorexant had limited effects on electroencephalographic power spectral density in contrast to three comparison insomnia treatments including zolpidem, gaboxadol, and trazodone. ${ }^{24}$ This information coupled with the pooled analysis of PSG data from the two 3-month trials of suvorexant use suggests that DORAs may improve sleep outcomes without changing the patient's neurophysiology. ${ }^{7,24}$ Another important finding related to sleep architecture is protection of response to salient stimuli in patients receiving DORAs compared with GABA-A receptor antagonists. In nonhuman primates, DORAs appear to protect one's ability to respond to important stimuli (eg, a fire alarm) while ignoring irrelevant stimuli (eg, bird chirping) compared with GABA-A receptor antagonists, such as eszopiclone and diazepam. ${ }^{5}$

A meta-analysis of suvorexant demonstrated that it did not increase suicidal ideation and/or behavior, events indicative of abuse potential, or motor vehicle accidents/ violations compared with placebo. ${ }^{14}$ However, the trials by Herring et $\mathrm{a}^{10}$ and Michelson et al included patients with primary insomnia as defined by Diagnostic and Statistical Manual of Mental Disorders, fourth edition, text revision and likely under-enrolled patients with depression and/or substance abuse disorders. ${ }^{12}$ A Phase I study of 36 patients demonstrated that the abuse potential of suvorexant is comparable to placebo; however, postmarketing data will be key in understanding its abuse potential in a larger, more diverse patient population..$^{21}$

Because suvorexant is the first in its class and only available as a branded medication, it is expensive (average wholesale price $[\mathrm{AWP}]=\$ 11.37$ per tablet or $\$ 341$ for 30 tablets of suvorexant $10 \mathrm{mg}$ or $20 \mathrm{mg}$ ) and is only available in one formulation. Therefore, patients cannot tailor the use of suvorexant based on their sleep patterns as is possible with other sleep agents that are supplied as immediate release tablets, extended release tablets, sublingual tablets, and spray formulations.

In summary, the beneficial effects of suvorexant likely outweigh any potential harm of the medication, and there are long-term (12 month) data with suvorexant to support its use as an agent for chronic insomnia. ${ }^{15}$ However, more real-world experience is needed with the medication to fully understand its safety and efficacy implications as the patient populations studied in clinical trials of suvorexant have largely been healthy patients with insomnia. It remains to be seen whether suvorexant will join or perhaps surpass traditional sleep agents as a first-line treatment for chronic insomnia.

\section{Conclusion}

Suvorexant, the first DORA to be approved for the treatment of insomnia, is a promising agent that offers a new option for patients seeking treatment of their insomnia. Studies that supported the approval of suvorexant demonstrated a favorable risk-benefit profile for most patients. Its mechanism of 
action, PK profile, and relative lack of rebound and withdrawal effects render suvorexant a rational choice for the treatment of chronic insomnia. Ongoing studies of suvorexant and head-to-head trials with other FDA-approved insomnia agents will assist in better defining the role of suvorexant in the treatment of chronic insomnia.

\section{Disclosure}

The authors report no conflicts of interest in this work.

\section{References}

1. Roth T. Insomnia: definition, prevalence, etiology, and consequences. J Clin Sleep Med. 2007;3(5 suppl):S7-S10.

2. Sateia MJ. International classification of sleep disorders-third edition. Chest. 2014;146(5):1387-1394.

3. Merck \& Co., Inc. Belsomra [Package Insert]. Whitehouse, NJ: Merck \& Co., Inc; 2014.

4. Krystal AD, Benca RM, Kilduff TS. Understanding the sleep-wake cycle: sleep, insomnia, and the orexin system. J Clin Psychiatry. 2013;74(suppl 1):3-20.

5. Uslaner JM, Renger JJ, Coleman PJ, Winrow CJ. A new class of hypnotic compounds for the treatment of insomnia: the dual orexin receptor antagonists. Orexin and Sleep. Sakurai T, Pandi-Perumal SR, Monti JM, editors. Cham: Springer International Publishing; 2015:323-338.

6. Jacobson LH, Callander GE, Hoyer D. Suvorexant for the treatment of insomnia. Expert Rev Clin Pharmacol. 2014;7(6):711-730.

7. Snyder E, Ma J, Svetnik V, et al. Effects of suvorexant on sleep architecture and power spectral profile in patients with insomnia: analysis of pooled phase 3 data. Sleep Med. 2016;19:93-100.

8. Gotter AL, Winrow CJ, Brunner J, et al. The duration of sleep promoting efficacy by dual orexin receptor antagonists is dependent upon receptor occupancy threshold. BMC Neurosci. 2013;14(1):90.

9. Sun H, Kennedy WP, Wilbraham D, et al. Effects of suvorexant, an orexin receptor antagonist, on sleep parameters as measured by polysomnography in healthy men. Sleep. 2013;36(2):259-267.

10. Herring WJ, Snyder E, Budd K, et al. Orexin receptor antagonism for treatment of insomnia: a randomized clinical trial of suvorexant. Neurology. 2012;79(23):2265-2274.

11. Herring WJ, Connor KM, Ivgy-May N, et al. Suvorexant in patients with insomnia: results from two 3-month randomized controlled clinical trials. Biol Psychiatry. 2016;79(2):136-148.
12. Michelson D, Snyder E, Paradis E, et al. Safety and efficacy of suvorexant during 1-year treatment of insomnia with subsequent abrupt treatment discontinuation: a phase 3 randomised, double-blind, placebo-controlled trial. Lancet Neurol. 2014;13(5):461-471.

13. Roehrs TA, Randall S, Harris E, Maan R, Roth T. Twelve months of nightly zolpidem does not lead to rebound insomnia or withdrawal symptoms: a prospective placebo-controlled study. J Psychopharmacol. 2012;26(8):1088-1095.

14. Kishi T, Matsunaga S, Iwata N. Suvorexant for primary insomnia: a systematic review and meta-analysis of randomized placebo-controlled trials. PLoS One. 2015;10(8):e0136910.

15. Citrome L. Suvorexant for insomnia: a systematic review of the efficacy and safety profile for this newly approved hypnotic - what is the number needed to treat, number needed to harm and likelihood to be helped or harmed? Int J Clin Pract. 2014;68(12):1429-1441.

16. Glass J, Lanctôt KL, Herrmann N, Sproule BA, Busto UE. Sedative hypnotics in older people with insomnia: meta-analysis of risks and benefits. BMJ. 2005;331(7526):1169.

17. McMillan JM, Aitken E, Holroyd-Leduc JM. Management of insomnia and long-term use of sedative-hypnotic drugs in older patients. CMAJ. 2013;185(17):1499-1505.

18. Sun H, Palcza J, Card D, et al. Effects of suvorexant, an orexin receptor antagonist, on respiration during sleep in patients with obstructive sleep apnea. J Clin Sleep Med. 2016;12(1):9-17.

19. Sun H, Yee KL, Gill S, et al. Psychomotor effects, pharmacokinetics and safety of the orexin receptor antagonist suvorexant administered in combination with alcohol in healthy subjects. $J$ Psychopharmacol. 2015;29(11):1159-1169.

20. Cui D, Cabalu T, Yee KL, et al. In vitro and in vivo characterisation of the metabolism and disposition of suvorexant in humans. Xenobiotica. 2016;46:1-14.

21. Schoedel KA, Sun H, Sellers EM, et al. Assessment of the abuse potential of the orexin receptor antagonist, suvorexant, compared with zolpidem in a randomized crossover study. J Clin Psychopharmacol. Epub 2016 May 31.

22. Kuriyama A, Honda M, Hayashino Y. Ramelteon for the treatment of insomnia in adults: a systematic review and meta-analysis. Sleep Med. 2014;15(4):385-392.

23. Winkler A, Auer C, Doering BK, Rief W. Drug treatment of primary insomnia: a meta-analysis of polysomnographic randomized controlled trials. CNS Drugs. 2014;28(9):799-816.

24. Ma J, Svetnik V, Snyder E, Lines C, Roth T, Herring WJ. Electroencephalographic power spectral density profile of the orexin receptor antagonist suvorexant in patients with primary insomnia and healthy subjects. Sleep. 2014;37(10):1609-1619.
Nature and Science of Sleep

\section{Publish your work in this journal}

Nature and Science of Sleep is an international, peer-reviewed, open access journal covering all aspects of sleep science and sleep medicine, including the neurophysiology and functions of sleep, the genetics of sleep, sleep and society, biological rhythms, dreaming, sleep disorders and therapy, and strategies to optimize healthy sleep. The manuscript

\section{Dovepress}

management system is completely online and includes a very quick and fair peer-review system, which is all easy to use. Visit http://www. dovepress.com/testimonials.php to read real quotes from published authors. 